\title{
INTEGRITY OF SUBSEA CONTROL UMBILICAL
}

\author{
Ramin Yasseri \\ MEng Student \\ Brunel University Uxbridge, Middlesex, UK
}

\author{
Sirous Yasseri \\ Chief Engineer \\ Safe-Sight Technology, Surbiton, Surrey, UK
}

\author{
Dr Bin Wang \\ Reader in Mechanical Engineering \\ Brunel University Uxbridge, Middlesex, UK
}

\begin{abstract}
Controlling subsea oil and gas production systems requires fibre optics, power cables and tubes for the transportation of chemical for injection and fluid for hydraulic controls. These are arranged in helical bundles in successive layers. Shaped thermoplastic fillers are used to separate components and to fill out voids in the cross section. If necessary, armour is added to provide strength for the dynamic loads. This paper describes the application of 3D finite element method to assess the structural Integrity of subsea umbilicals, including the interaction between the constituent elements. It is suggested to uses a global analysis to determine the internal forces and local analyses (sub-modelling) for detailed stress evaluation. Abaqus is used for this purpose, due to its capability with large size problems under severe discontinuities due contact conditions. This paper also discusses the umbilicals interaction with seabed. The focus of this paper is on the fatigue life calculation.
\end{abstract}

\section{INTRODUCTION}

Umbilicals are a long, flexible construction - its length can be from a few kilometres to more than $200 \mathrm{~km}$. Umbilicals consist of tubes, cables, armouring, fillers and wrapping which are contained within a protective sheath. The multiple functions that are demanded of umbilicals in harsh environments require high reliability and dependability, as repairing or replacing them is an expensive activity.

The primary function of a subsea umbilical is to provide link between facilities on a host platform to the subsea production equipment on the seabed. The number of power cables and tubes depends on the requirement and sparing policy. Combining all utility lines into bundles enables fast installation by reeling.

An umbilical is either designed for dynamic or static conditions [4]. A static umbilical is installed along the seabed or trenched and once installed the fluid conduits are only subjected to internal and hydrostatic pressure. This type of umbilicals is used to connect subsea equipment, or provide long distance tiebacks to onshore control centres, or fixed platforms. A dynamic umbilical is used to link floating topsides to subsea equipment and is designed to withstand high pressure fluid containment and a high tensile load with fluctuations generated by the vessel motion as well as waves and currents (Figure 1). In deeper water, the strength of umbilical becomes an important factor, as installation loads are greater for both static and dynamic umbilicals, causing high tensile load coupled with low radius bending, which could endanger the umbilical's integrity.

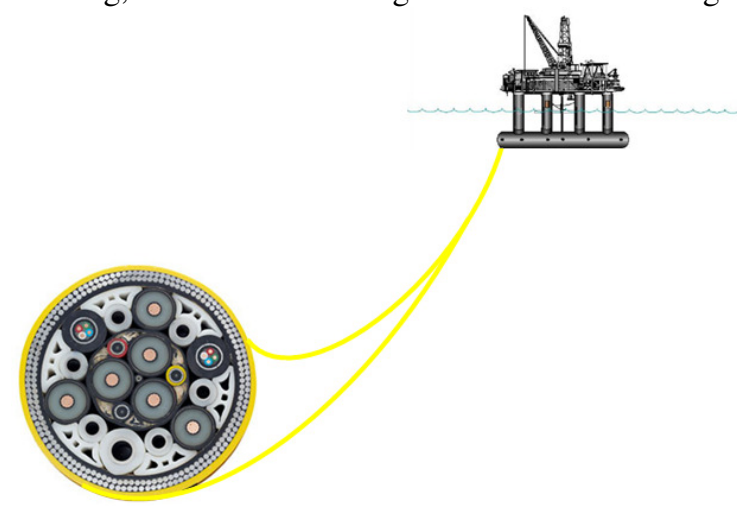

Figure 1: A dynamic umbilical connecting a floater to the subsea facilities

Umbilicals are line-like structures; namely one of their dimensions (the cross-section) is vastly smaller than their length and hence they are flexible. They may undergo large displacement without straining. The factor that differentiates umbilicals from each other, is the composition of their cross sections. Flexible pipes are made of several layers of steel and thermo plastics, while control umbilicals are a number of small tubes, electrical cables and fibre optic bundled together within a sheath cover. If one ignores what is happing between various 
layers making up a flexible umbilical, then their global behaviour can be studied in a similar fashion.

Components are generally bundled together helically which adds to the overall strength and flexibility. In multi-layered umbilicals the layers are usually contra rotated. Umbilicals are not bonded together, but kept apart using thermo-plastic fillers. Helical winding makes the response which is known as stickslip behaviour ([19]). When loadings are not large enough to overcome the shear friction at the contact points, the components will stick together. The shear resistance at the interfaces are governed by the friction coefficient, and the reaction forces at the interfaces generated by the tension and torsion exerted on the umbilical. When the interface shearing resistance is exceeded then components slip and move from the compressive side towards the tensile side of the umbilical.

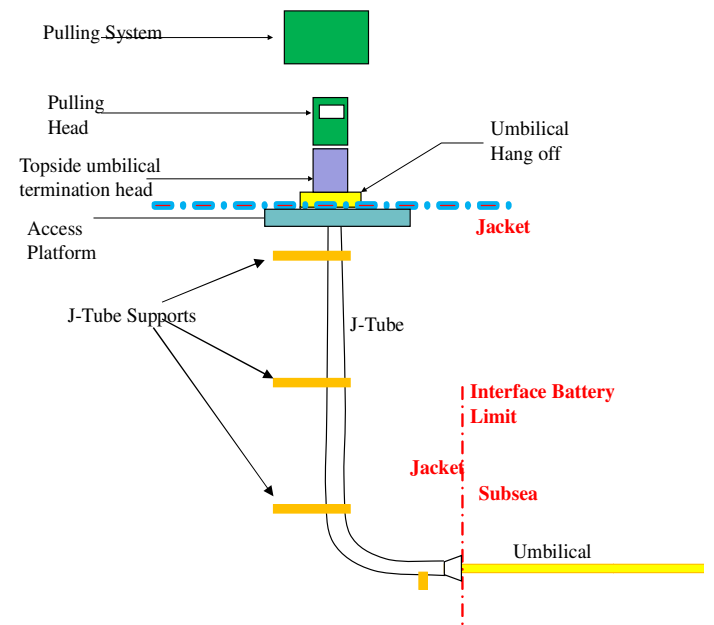

Figure 2: Connection of umbilical to the topside in fixed platform system.

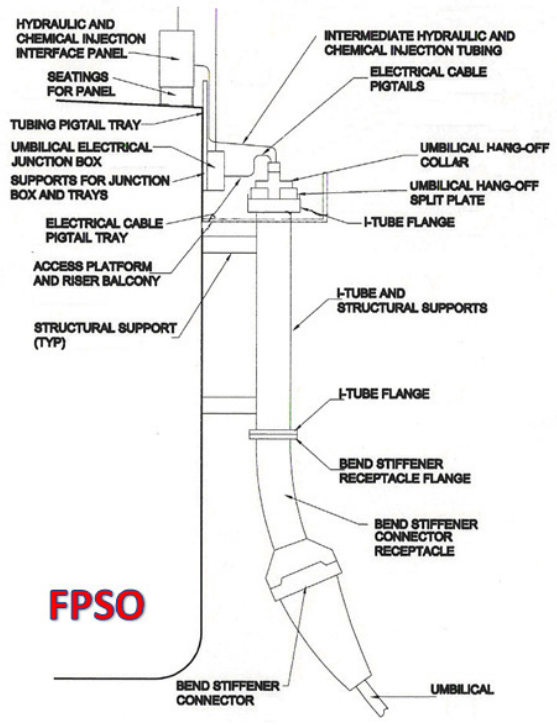

Figure 3: Umbilicals connection to an FPSO - From a project re-sources.
If the dynamic section hangs freely between the vessel and the seabed, the configuration is known as a free-hanging catenary. Different installation configurations that provide support for the umbilical in the water column may also be used, such as lazy wave, pliant wave, reverse pliant wave and steep wave. In general, the complexity and severity of the requirements for dynamic umbilicals increase with increasing water depth- see [21] for power cables, and [12] for arctic umbilicals.

In the case of a fixed platform, the umbilical is usually installed and supported in a J-tube or I-tube which runs from the surface to the seabed (Figure 2 and Figure 3 ). The section of the umbilical installed in either type of tube is considered to be static.

\section{PERFORMANCE REQUIREMENTS}

A performance specification establishes functional requirements under various operating conditions. Compliance with performance requirements can be measured by analytical methods (the purpose of this paper) or standard test methods with defined acceptance criteria. Unless it is permitted, it is assumed that the component materials used, comply with industry standards. The performance specification establishes measureable and enforceable criteria in contract documents, to be met by the supplier.

There are several codes of practice giving recommendations on the design of umbilicals. The most popular among them are [2], [8], [19] and [25] Standard. The functionality must be checked under the following loading conditions:

- $\quad$ Functional loads: These are loads acting during manufacturing, installation and operation, including loads that can act in still water cases. Wind, wave and current are excluded from this category.

- $\quad$ Environmental loads: loads applied to the umbilical from the environment, i.e. wind, wave, current and vessel motion

- $\quad$ Accidental loads: which are induced by undesirable events, such as incorrect operation, abnormal condition and technical failure. Examples of such loads are dropped objects, anchor drop and anchor hooking as well as hazards due to fishing activities. However this type of loads is condition dependent.

In addition to the above accidental loads, in certain geographical region, Geohazards could impose a threat. Such hazards include, seismic ground shaking, fault crossing, slope failure, unstable ground and turbidly flow.

Codes require the above loads to be combined in certain fashion. For example ISO-13628-5 [19] recommends the following load combination: 
- $\quad$ Normal operation: This applies to the permanent operational state of the umbilical, taking into consideration functional and environmental loads.

- Abnormal operation: This applies to the permanent operational state of the umbilical taking into account functional, environmental and accidental loads.

- Temporary conditions: This applies to temporary conditions, such as installation, retrieval, pressure testing and other intermediate conditions prior to permanent operation. ISO-13628-5 [19] requires the fatigue life to be studied under the following conditions:

- Direct wave loading as well as wave induced host motion.

- Slow drift host motions including variation of mean position

- VIV response of the umbilical under steady current conditions

- $\quad$ Cyclic loading during fabrication and installation

- $\quad$ Cyclic loading due to operation of the umbilical.

In general, similar failure modes as for the pipelines is observed for the static umbilical and failure mode similar to deep-water riser for the dynamic umbilical and hence recommendations of pipeline and rise codes are pertinent.

\section{ON BOTTOM STABILITY}

On-Bottom Stability Design of Submarine Pipelines is applicable for determining umbilicals lateral stability. DNVRP-F109 [9] method is based on an equilibrium balance of forces acting on the cable (Figure 4). The dynamic simulation also incorporates random wave generation and hydrodynamic forces and soil modelling.

The difference between size of an umbilical and a typical pipeline differentiates them. Umbilicals will generally sit further into the turbulent boundary layer than pipelines because of their size difference. This introduces a potential source of error into the calculation of forces, due to the steady flow velocity being a key input parameter.

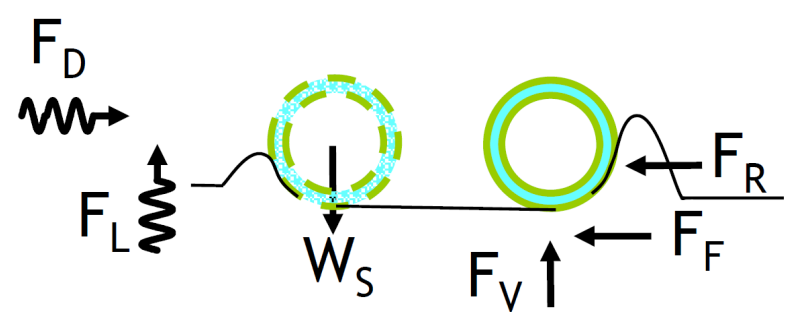

Figure 4: Wave forces acting on an un-trenched umbilical.

\section{FREE SPAN}

DNV-RP-F105 [8] is used for assessing the likelihood of Vortex-Induced Vibration (VIV) for steel pipelines, and may be modified in order to be applicable to subsea transmission cables. VIV can have serious consequences for the fatigue life. The allowable span length is determined by comparing the vortex shedding frequency at a range of possible flow rates with the natural frequency of the umbilical. Umbilicals have significant internal damping, and the damping effect of the surrounding fluid can also significantly alter the vibration characteristics

Finite element analysis is used to determine the effect of spans along the route. The umbilical is modelled as laid on a flat condition and in the next step it is gradually lowered onto a 3D seabed with bathymetry data of the route.

\section{GLOBAL-LOCAL ANALYSIS}

Many researchers (e.g. see [7], [17], [18], [20], [21], [23], [26], [27]) investigated flexible marine structures, such as flexible pipes, deepwater risers and umbilicals.

Analysing of umbilicals using solids or shells elements leads to a prohibitively large model with many degrees of freedom. Thus, the analysis is carried-out in two levels; Figure 5. One way to alleviate this problem is to use global-local modelling approaches, one method of which is often referred to as "submodelling". The commercial FE package ABAQUS includes a sub-modelling technique. Sub-modelling is not the only reduction technique, but by far the most popular one.

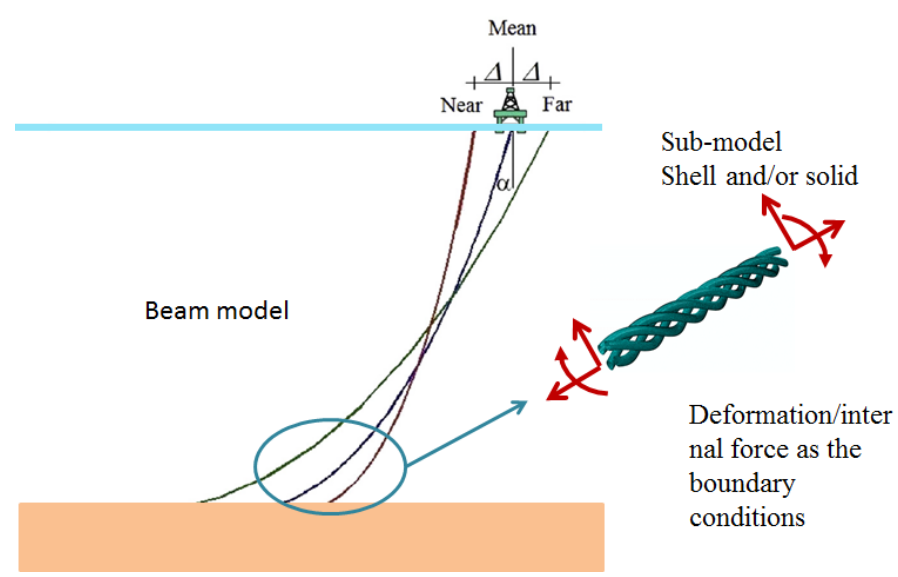

Figure 5: Global model and the sub-mode

Sub-modelling employs two models. The global model represents the entire structure and contains a coarse representation of the domain of interest (for example using general beam elements to represent a complex cross section). However, the entire global model is sufficiently refined to accurately calculate the displacement solution on the boundary of the domain of interest. Subsequently, the solution obtained from the global model along the boundary of interest is applied as a displacement boundary condition on the sub-model. In Abaqus transfer of data (which may require interpolation) is performed automatically by the software. However, with some 
forethought in modelling extracting displacement from the global model and applying to the local model would be as easy.

The design process begins with the definition of the functional requirements, including the configuration and geometric parameters, installation and operation conditions, floater data, environmental conditions, etc. The first step is the conceptual design, which involves the number of tubes, power cables and fibre optics, choice of materials for each component, inner \& outer sheaths, and armour layer.

In this approach, the global analysis of the umbilical is carriedout using pipe elements or beam elements (general Section in Abaqus) for the general section. These elements will capture the dynamic and nonlinear effects in a simple and efficient manner, provided the mesh is good enough. It is necessary that the equivalent mechanical properties $(E A, E I$, and $G J)$ of the umbilical are correctly determined. These can be obtained by a combination of testing and a 3-D finite element of a length of the umbilical. The compatibility "EA, EI and GJ" with shell/solid sub-model verified my manual calculations as well using the sub-model to replicate these parameters. In the Global analysis the bend restrictor area is modelled with still smaller size elements in order to capture changes in stiffness.

The global analysis yields the displacements and stresses resultants (axial force, bending and torsional moments) along the umbilical length. These results are good enough to check the umbilical strength under extreme conditions [30]. However, the use of beam elements does not allow the direct computation of stresses and strains of each component of the umbilical for the fatigue calculations. More detailed information is required for the fatigue life determination. Thus, the stress resultants and displacements calculated in the global analysis at critical sections are used as input for local analysis (sub-modelling). These critical sections are at the touch down zone, and the point where the umbilical is connected to the vessel; and perhaps at the mid-height buoy if it is used. The local analysis is carriedout using refined shell or solid finite element models, as these elements allow the computation of stresses and strains for each component, as required by the fatigue analysis procedure using S-N curves.

The sub-model is a highly refined model of the domain of interest. The primary assumption of sub-modelling is that the structural details of the sub-model do not significantly affect the solution in the global model. In most practical applications, there are no a priori methods for determining the validity of this assumption. It is therefore left to the analyst to determine the validity of this assumption, based on prior experience.

It should be remembered that both global and local analyses used in this study are dynamic and performed by the direct integration capability of Abaqus/Standard
The time-dependent fidelity of the boundary conditions that are applied to the sub-model is known to affect the results. To eliminate this effect the sub-model consists of the detailed geometry of the umbilical between two nodes of the global model, in order to avoid interpolation of displacement. The time histories of displacements of these nodes from the global analysis were applied to the local model. Additionally, operational loads (depending on the location of sub-model were also applied to achieve the required fidelity.

\section{DESIGN DATA}

Many situations must be considered to ensure the long term integrity of umbilicals. These include: on bottom stability, spanning, extreme condition, unstable ground, VIV, dropped objects, fatigue, vessel motion and so on.

Vessel motion is a major component of the loading, since it can induce most of the fatigue damage to the umbilical. The motion of a vessel can be specified in a number of ways: directly by a time history motion data or by specifying Response Amplitude Operators (RAOs) for each of 6 degrees of freedom (surge, sway, heave, roll, pitch and yaw - Figure 6), or indirectly by giving first order wave load RAOs or Second order wave drift QTFs (Quadratic Transform Functions), or a combination of both.

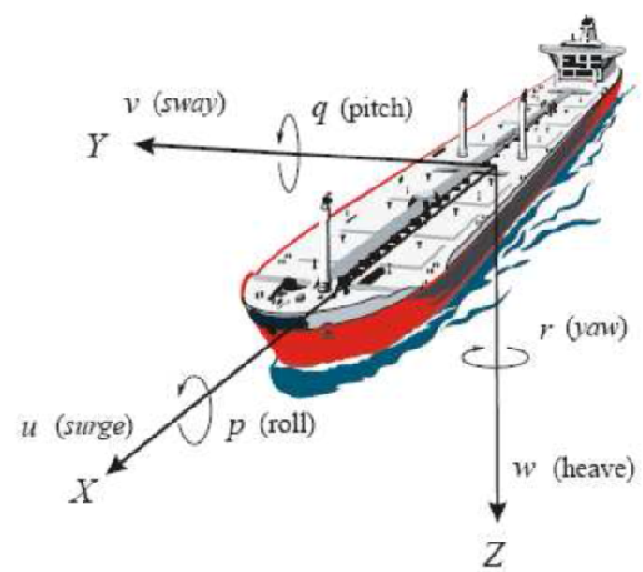

Figure 6: Definition of surge, sway, heave, roll, pitch and yaw, modes of motion in body-fixed frame. (From internet resources)

Vessel motions in waves are commonly defined by displacement Response Amplitude Operators (RAOs). Each displacement RAO consists of a pair of numbers that defines the vessels response, for one particular degree of freedom, to one particular wave direction and period. The two numbers are amplitude, which relates the amplitude of the vessel motion to the amplitude of the wave, and a phase, which defines the timing of the vessel motion relative to the wave. RAOs can be obtained either from model test or via computational methods. 
All vessel motions are applied to the umbilical at the point of attachment to the vessel. This is a major critical fatigue region, which occurs directly below the attachment point. The applied motion at the top of the umbilical is transferred down the length of the umbilical and is converted to bending, above the point at which the umbilical touches down on the seabed, the touch down zone (TDZ). This is another critical fatigue region. The fatigue damage is highest at the touch down zone. Assumption can be made regarding the effect of seabed material affect the fatigue life in the TDZ; for example, a rigid seabed can give high levels of conservatism when compared to an elastic seabed ([15] and [28].

The operational loads are the density and pressure of content and the environmental effects. The strength of an umbilical is checked for extreme cases. In extreme storm events the vessel motions may be severe enough to cause compression and buckling in the touch down point region of a simple catenary umbilical. Response of umbilicals is non-linear and time dependent.

For the design of offshore structures a "design" wave is defined which has 100 year return period. The term 'regular' refers to unidirectional train of waves with constant amplitude and frequency and hence constant length. If the steepness (= length/amplitude) is sufficiently small, the waves are said to be linear or Airy waves.

Ocean waves are random and complex and may be idealised as a large number of wavelets of different heights, periods and directions. They can effectively be modelled in terms of regular wave trains, combining in random phase. An important advantage of such modelling is that the complete description of the wave motions including surface kinematics for the regular components can be linearly superposed to provide corresponding complete descriptions of the combined wave motion. The random nature of the surface of the oceans means that it can only be quantified statistically. The statistics do not change very much over an interval of about 3 hours and the sea-state is characterized by significant wave height and mean zero-crossing period, as defined below, and sometimes by peak period (Tp).

The significant wave height denoted by $H s$ is a common representative height for a sea-state. Originally, it was defined as the mean value of the highest one third of a sample of the sea-state wave heights. According to modern definitions, $H s$ is defined in terms of the variance of the sea surface elevation.

ABAQUS/AQUA requires the input sea-states to be given in the form of waves. Thus a sea-state needs to be decomposed into several sinusoidal wave components identified by 3 parameters i.e. amplitude, period and phase angle.

The mean zero up-crossing periods denoted by $T_{z}$ is another characteristic of a sea-state. The customary practice in wave analysis is to utilize either the zero-up-crossing method or zerodown-crossing method as the standard techniques for defining wave ([3] and [11]).

This study uses Pierson-Moskowitz (PM) energy density spectrum which is wildly used for characterising waves in the open sea ([3]). There are several expressions for PM energy density spectrum. The following formula of the PM spectrum ([3]), which depends on the sea-state representing parameters i.e. $H_{s}$ and $T_{z}$, has been used in this study to decompose a seastate into several sinusoidal wave components [3].

$S\left(f_{i}\right)=\left(\frac{A}{f_{j}^{4}}\right) \times e^{-\frac{B}{f_{j}^{4}}} \quad 0<f_{j}<\infty$

Where

$A=\frac{H_{S}^{2}}{4 \pi T_{z}^{4}}, B=\frac{1}{\pi T_{z}^{4}}, f_{j}$ is the frequency of $j t h$ wave component $\left(H_{z}\right), H_{s}$ is the significant wave height $(m), T_{z}$ is the mean zerocrossing period of a sea-state $(s)$.

Zeroth, first and second spectral moments are obtained from [3]:

$m_{0}=\frac{A}{4 B} ; m_{1}=\frac{0.306 A}{B^{\frac{3}{4}}} ; m_{2}=\frac{\sqrt{\pi}}{4} \frac{A}{\sqrt{B}}$

When decomposing an irregular sea state to be used in the analysis, one technique is to divide the wave spectrum into $\mathrm{N}$ columns of width $\Delta \omega$ (or in term of frequency $f_{j}$ ), as shown in the upper part of Figure 7. The lower half of the figure shows how the wave components are summed up to give the resulting irregular sea. Figure 8 shows another way of visualizing another way to visualize the transition from the wave spectrum in the frequency domain to the irregular wave elevation in the time domain


Figure 7: An irregular sea state is decomposed into several regular waves, using a spectrum. The arrows indicate the quasi-random wave heights (from [16]). This figure waves are presented in terms of circular frequency, $(\omega=2 \pi f)$.

It was assumed the Pierson-Moskowitz (P-M) spectral formulation represents the sea-state. Each sea-state was 
decomposed into 30 sinusoidal components having its own amplitude, frequency and phase angle. The phase angles were generated randomly between 0 and 360 degrees. Then for each sea-state the range of frequency (bandwidth), i.e. zero to where the spectrum approaches zero, was determined and divided into 30 subintervals. The frequency of each subinterval end point was selected as each component frequency. Then, given the frequency of the component, its amplitude was calculated using the following formula:

$a_{j}=\sqrt{2 S\left(f_{j}\right) \times \Delta f_{j}}$

Where

$a_{j} \quad=$ the amplitude of $\mathrm{j}^{\text {th }}$ component,

$f_{j}=$ the frequency of $\mathrm{j}^{\text {th }}$ component,

$S\left(f_{j}\right)=$ energy density given by Eq. (1),

$\Delta f_{j}=\left(f_{j+1}-f_{j-1}\right) / 2$

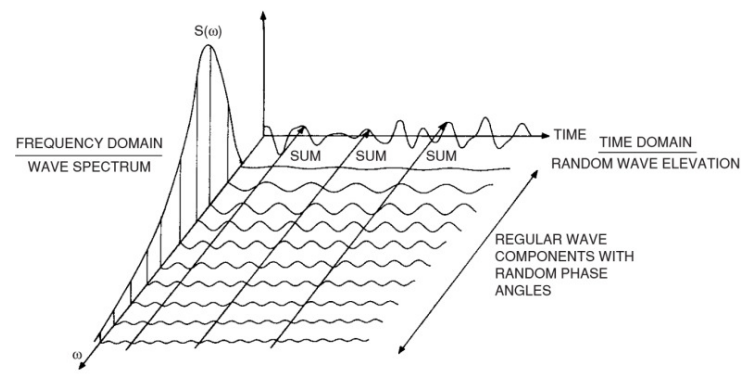

Figure 8: The connection between the frequency-domain and time-domain representation of waves (From [11])

Table 1: Simplified Scatter diagram for North Atlantic Sea conditions

\begin{tabular}{|l|l|l|}
\hline \multicolumn{2}{|l|}{$\begin{array}{l}\text { Sea-State } \\
\text { Definition }\end{array}$} & \multicolumn{2}{l}{$\begin{array}{l}\text { Sea State } \\
\text { Occurrence } \\
(\%)\end{array}$} \\
\cline { 1 - 2 } Tzu (sec) & Hs (m) & \\
\hline 1.95 & 0.3 & 7.2 \\
\hline 3.34 & 0.88 & 22.2 \\
\hline 4.88 & 1.88 & 28.7 \\
\hline 6.42 & 3.25 & 15.5 \\
\hline 7.96 & 5.00 & 18.7 \\
\hline 9.75 & 7.50 & 6.1 \\
\hline 12.07 & 11.50 & 1.2 \\
\hline 13.32 & 14.0 & 0.2 \\
\hline
\end{tabular}

The P-M wave height spectrum with parameters related to open seas and a North Atlantic wave scatter diagram has been employed in this study (see Table 1).

\section{CASE EXAMPLE}

This section details the application of the design procedure outlined above to an umbilical in $500 \mathrm{~m}$ of water. Figure 9 shows the static section of the umbilical and Tables 2 and 3 give the rest of data.

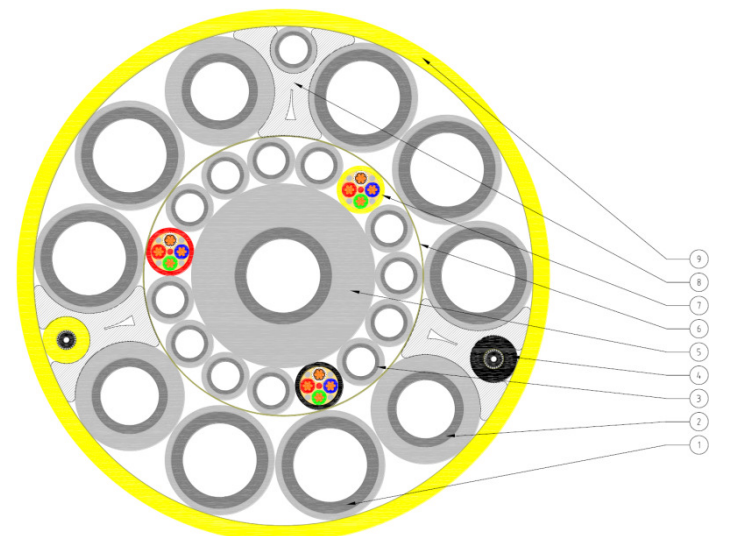

Figure 9: The static part of the example umbilical. From a project files.

Table 2: Composition of the static part of umbilical

\begin{tabular}{|c|c|c|c|c|}
\hline Item & QTY & Description & $\begin{array}{l}\text { Nominal } \\
\text { thickness } \\
\mathrm{mm}\end{array}$ & $\begin{array}{l}\text { Nominal } \\
\text { Diameter } \\
\mathrm{mm}\end{array}$ \\
\hline 1 & 8 & $\begin{array}{l}1.25 " \text { SD steel tube, } \\
900 \text { bar , sheathed }\end{array}$ & 5.08 & 42.16 \\
\hline 2 & 3 & $\begin{array}{l}\text { 1" SD Steel tube, } 900 \\
\text { bar, sheathed }\end{array}$ & 3.85 & 33.1 \\
\hline 3 & 13 & $\begin{array}{l}\text { 1/2" SD steel Tube, } 900 \\
\text { bar, Sheathed }\end{array}$ & 1.77 & 16.24 \\
\hline 4 & 2 & $\begin{array}{l}\text { Fibber Optic Element, } \\
24 \text { SM fibbers, } \\
\text { armoured }\end{array}$ & & 20.2 \\
\hline 5 & 1 & Centre tube sheath & & 18. \\
\hline 6 & & Wrapping & 0.32 & \\
\hline 7 & 3 & $\begin{array}{l}\text { Electrical Qua } \\
16 \mathrm{~mm}^{\wedge} 2,1 \mathrm{kV}\end{array}$ & & \\
\hline 8 & 3 & Profiled Filler & & \\
\hline 9 & 1 & HDP Outer Sheath & 6.8 & $\sim 230$ \\
\hline
\end{tabular}

Table 3: Mechanical properties of the example umbilical

\begin{tabular}{|l|l|l|}
\hline Parameter & Parameter & Unit \\
\hline $\begin{array}{l}\text { MHT (utilisation factor of 100\% } \\
\text { SMYS) }\end{array}$ & 1996 & {$[\mathrm{KN}]$} \\
\hline $\begin{array}{l}\text { Min. breaking load (No bending, } \\
\text { 100\% UTS) }\end{array}$ & 3593 & {$[\mathrm{KN}]$} \\
\hline MBR (elastic limit) & 6,69 & {$[\mathrm{~m}]$} \\
\hline Axial stiffness & 1376 & {$[\mathrm{MN}]$} \\
\hline Bending stiffness & 243 & {$[\mathrm{kNm} 2]$} \\
\hline Torsion stiffness & 275 & {$[\mathrm{kNm} 2]$} \\
\hline
\end{tabular}

The umbilical is installed in $500 \mathrm{~m}$ water (Figure 10) and connected to an FPSO. The far end is connected to a subsea cluster about 8 kilometers away. The umbilical make-up is the same along the length, except the about $1000 \mathrm{~m}$ (the dynamic 
part), which is connected to the vessel, has extra armor to achieve the required strength and fatigue life.

The analysis was conducted in a time domain covering 3 hours. To facilitate the derivation of spectral properties from the time series, the response was computed for at least 2048 (multiples of 1024- See [30]) sampling points for each location on the umbilical.

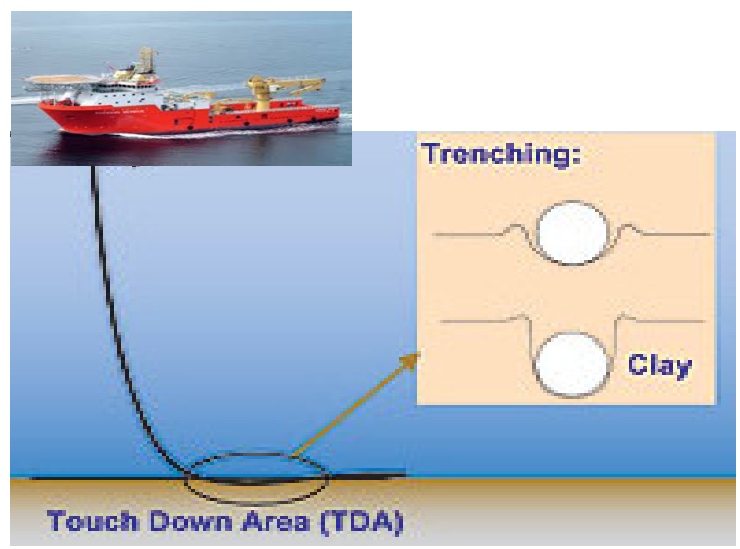

Figure 10: The umbilical configuration including a depiction of the touchdown zone.

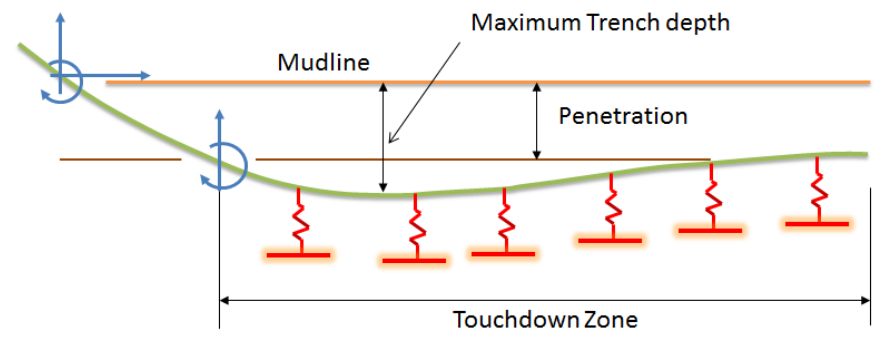

Figure 11: Touchdown zone as a bed of non-linear springs; see also [15] and [28].

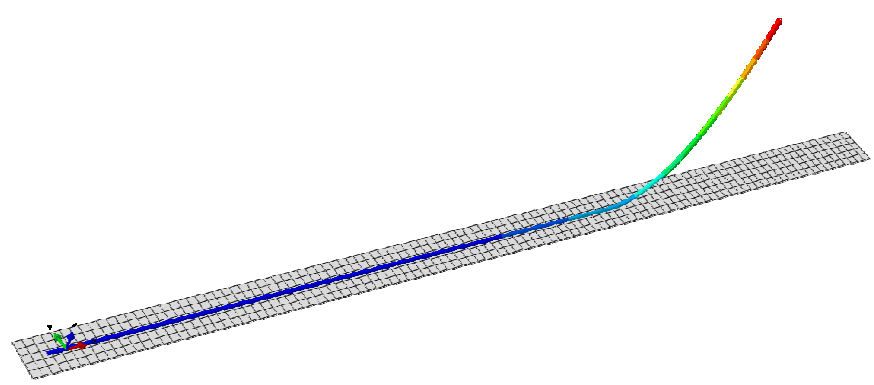

Figure 12: The global model

Various ideas for the modeling of the touchdown zone were explored (Figure 11). Assumption made regarding the effect of seabed material affect the fatigue life in the Touch down Zone
(TDZ). For instance, a rigid seabed can give high levels of conservatism when compared to an elastic seabed.

The umbilical was modeled on the seabed for the global analysis. In the first step of a multi-step analysis the self-weight was applied and a non-linear static analysis was performed to determine the equilibrium position. In the next step one end of the umbilical was lifted and brought it to the vessel mean position. The third step was a direct integration non-linear time domain analysis in which the umbilical was exposed to the effect of wave loading calculated by Morrison equation in ABAQUS. Airy wave theory was used for this analysis, as it is considered to be accurate for low amplitude waves in deep water. For each sea-state, the sea surface was presented using a large number of Airy waves. ABAQUS/AQUA [1] software was used to analyze the umbilical for all sea-states given in Table 1 .

The geometry and effective axial force $\left(\mathrm{N}_{\mathrm{eff}}\right)$ were also computed by the catenary solver from the effective weight $\left(\mathrm{w}_{\mathrm{e}}\right)$, top angle with the vertical $(\alpha)$, and sea depth for the mean configuration. This was checked against Abaqus results. Figure 12 shows the global model.

MATLAB was utilised to yield the wave amplitudes and wavelengths of the components representing the required sea state based on a Pierson-Moskowitz spectrum. Table 4 gives an example of the wave components for one sea state. The number of wave components has been limited to 30 components. The phase information was generated randomly [29].

\section{Table 4: Hydrodynamic Coefficients}

\begin{tabular}{|l|l|}
\hline $\begin{array}{l}\text { Hydrodynamic } \\
\text { Coefficients }\end{array}$ & Value \\
\hline $\begin{array}{l}\text { Drag Coefficient, } \\
\text { Normal }\end{array}$ & 0.7 \\
\hline Drag Coefficient, Axial & 0.0 \\
\hline Added Mass, Normal & 1.0 \\
\hline Added mass, Axial & 0.0 \\
\hline
\end{tabular}

Umbilical is a slender structure, hence Morison's equation is applicable. The basic assumption of Morrison method is that the diameter of cylinder $\mathrm{D}$ compared with wavelength $\mathrm{L}$ is small, $\mathrm{D} / \mathrm{L}<0.2$, hence the cylinder cannot affect the wave field. Abaqus uses Morrison's equation [3]. The hydrodynamic coefficients used for the riser are summarized in Table 4.

This form of simulation is subject to approximations and limitations that should be recognised. Limitations are introduced by the fact that the method adopted for the decomposition of the spectrum into wave components uses deterministic wave amplitudes fixed by the spectral values at equal frequency intervals. Consequently the randomness of the 
process is undermined and the wave signal may repeat itself at regular intervals. The approximation relates to the fact that only 30 components are used, increasing the likelihood of missing the contributions of a natural mode to the total response due to the greater spacing between the wave components. On the other hand, if wave components are close to a natural frequency then the resulting response would be conservative in comparison to using more wave components with lower amplitudes. More detail can be found in reference [30].

Table 4: A sample of Decomposition of a sea-state characterized by $H s=1, T z=3$

\begin{tabular}{|c|c|c|}
\hline Amplitude & Period & Phase angle \\
\hline (m) & (s) & (deg) \\
\hline 0 & 27.4 & 3342.0465 \\
\hline 0 & 13.7 & 283.2099 \\
\hline 0 & 9.14 & 218.4633 \\
\hline 0.0135 & 6.86 & 174.9537 \\
\hline 0.1006 & 5.49 & 320.8676 \\
\hline 0.1603 & 4.57 & 274.3549 \\
\hline 0.1619 & .3 .9 & 2164.3284 \\
\hline 0.1404 & 3.43 & 6.6613 \\
\hline 0.1159 & 3.05 & 295.7066 \\
\hline 0.0944 & 2.74 & 160.0932 \\
\hline 0.077 & 2.49 & 221.5557 \\
\hline 0.0634 & 2.29 & 285.0973 \\
\hline 0.0527 & 2.11 & 331.8527 \\
\hline 0.0442 & 1.96 & 265.7546 \\
\hline 0.0374 & 1.83 & 63.4558 \\
\hline 0.032 & 1.71 & 146.0542 \\
\hline 0.0276 & 1.61 & 336.7691 \\
\hline 0.024 & 1.52 & 330.0856 \\
\hline 0.021 & 1.44 & 147.6973 \\
\hline 0.0185 & 1.37 & 321.7138 \\
\hline 0.0164 & 1.31 & 20.8409 \\
\hline 0.0146 & 1.25 & 127.0325 \\
\hline 0.0131 & 1.19 & 292.7399 \\
\hline 0.0118 & 1.14 & 3.5501 \\
\hline 0.0107 & 1.1 & 50.0007 \\
\hline 0.0097 & 1.06 & 72.9955 \\
\hline 0.0088 & 1.02 & 71.5398 \\
\hline 0.008 & 0.98 & 217.3653 \\
\hline 0.0073 & 0.95 & 97.9877 \\
\hline 0.0068 & 0.91 & 71.5731 \\
\hline
\end{tabular}

The phase angle was genet red using random number generation in MATALB. True randomness cannot be achieved. Table 4 shows only one realisation of the phase angle. Other phase angles were also tested before setting on one [29].
The modelling of the sub-model starts with building a part in Abaqus-CAE whose cross section is exactly as shown in Figure 9. After saving the cross section of this part as a sketch (Figure 13), the building of the part is abandoned. This sketch gives all components in their exact location. Some components (e.g. fillers and tube's protective sheaves, cables and fibre optic) must be modelled as solid, while tubes can be modelled as shells. Avoiding modelling all components in solid also reduces the number of degrees of freedom. It was further assumed that if the sub-model is short ( $5.0 \mathrm{~m}$ here), then the curvature may be ignored, hence the number of different geometries to be built is reduced. This assumption is valid away from TDZ and bends restrictor area. However, models with actual curvature can be built by applying displacement to a model built as discussed and then the coordinates of deformed geometry is used as a new model.
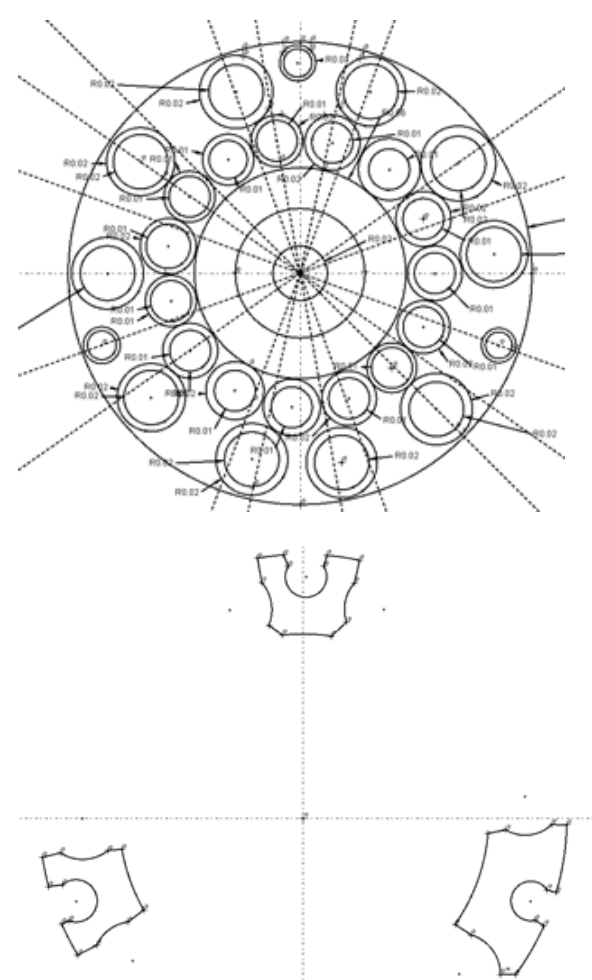

Figure 13: Sketch of the cross section. The top figure shows all components except the fillers. The bottom figure shows a sketch that was built for fillers. Abaqus/CAE

For this study, all tubes including the external protective sheath were modelled as shell and the rest as solid and all of them were created by extrusion with a given depth and twist (pitch). Although, the entire model can be built by calling up the sketches prepared earlier, a better policy is to create components (parts in Abaqus) one at a time.

Start creating a part by defining a size for the drawing and if the part is created by extrusion etc. and if it is solid or shell. Call up 
the previously generated sketch and delete irrelevant section and extruded the remaining lines (component of interest). All components within the umbilical can be built this way. In assembly module they are brought together. Due to using the same sketch for all of them, parts are in correct location. Such modelling approach, allows meshing each part individually and assigning its property also individually.

If the assumption of curvature has little influence, then the difference between various sub models is just the displacement histories at their boundaries. Since the length of the sub-model was divisible by the length of elements of the global model, the sub-model boundary coincides with the global nodes, thence no interpolation is need.

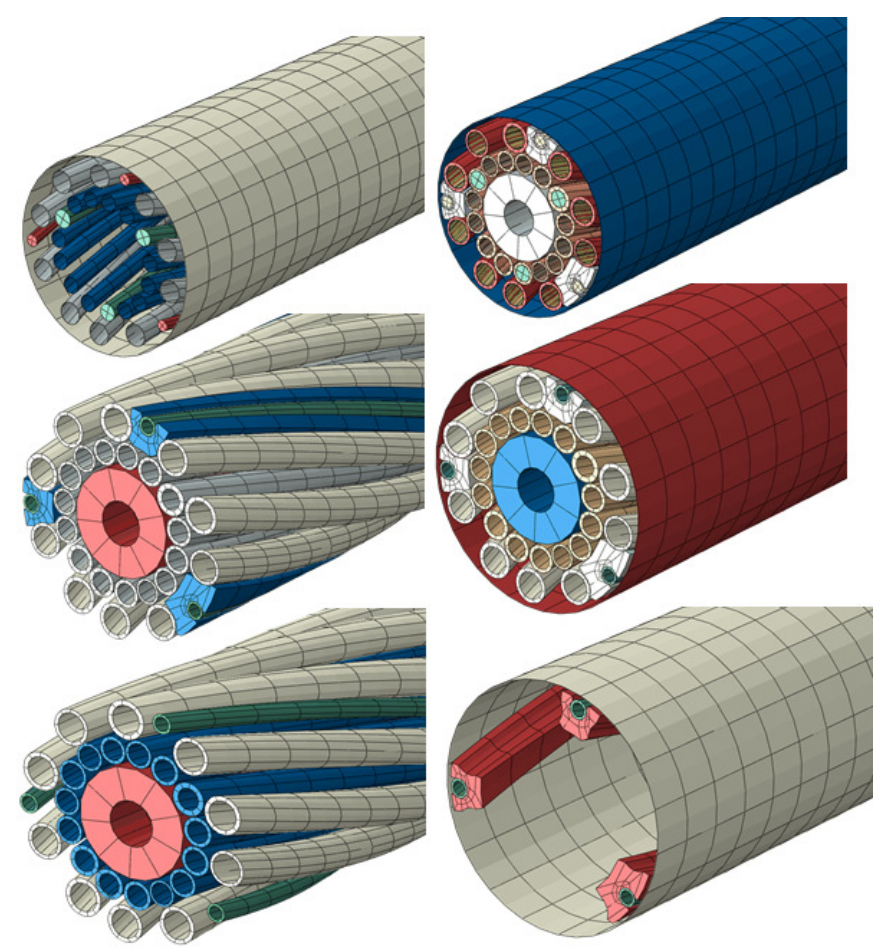

Figure 14: this figure shows the arrangement of example umbilical.



Figure 15: The geometry of the sub-model. Note that the model is a combination of solid and shell elements.
Figure 14 shows various view of the sub-model with some components removed for clarity. Figure 15 shows the entire model. While, Figure 16 shows only the major components (steel tubes) including the outer sheath.

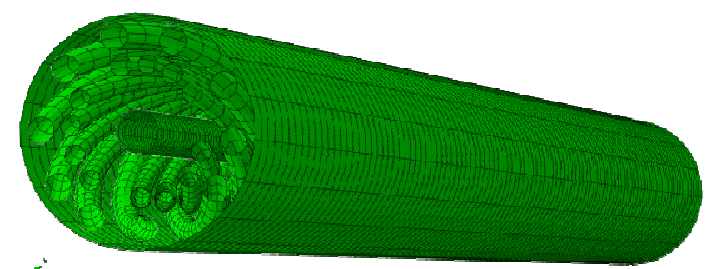

Figure16: The sub-model with filler material removed for the sake of clarity.

The global analysis of the models was carried out using nonlinear direct integration dynamic analysis capability of Abaqus/Standard [1]. The general contact in Abaqus was used. The penalty method was used for the tangential behavior (friction coefficient 0.45) and "hard" contact for normal behavior. In addition, the add-on option Abaqus/Aqua was used. It contains optional features that are specifically designed for the analysis of beam-like structures installed underwater and subject to loading by water currents and wave action

Results of global analyse were mapped onto the sub-models of various sections of the umbilical. Except for the vessel connection point and the TDZ sub-models look alike. Table 5 gives some details of the two models.

Table 5 Details of the models

\begin{tabular}{|l|l|l|l|l|}
\hline \multicolumn{2}{|l|}{ Model } & $\begin{array}{l}\text { Element } \\
\text { type }\end{array}$ & $\begin{array}{l}\text { Characteristic } \\
\text { length mm }\end{array}$ & $\begin{array}{l}\text { Number } \\
\text { of } \\
\text { elements }\end{array}$ \\
\hline \multirow{2}{*}{ Global } & Seabed & R3D4 & 500 & 12852 \\
\cline { 2 - 4 } & Umbilical & Pipe32 & 200 & \\
\hline \multirow{2}{*}{ Local } & All tubes & S8R & 50 & 228388 \\
\cline { 2 - 4 } & Others & C3D20R & 5 & \\
\hline
\end{tabular}

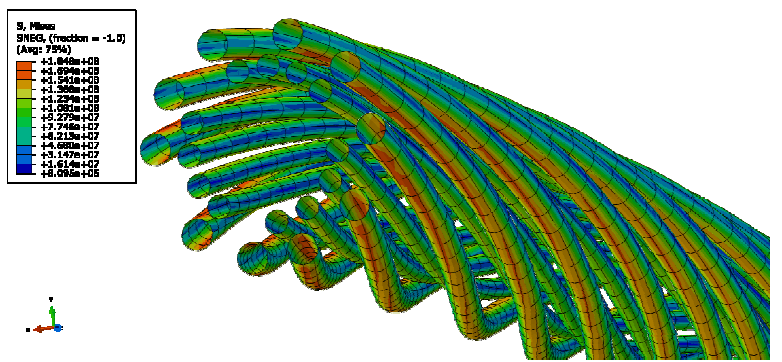

Figure 17: One frame of the time domain analyses results for the outer tubes.

The process of dynamic analyses for several sea sate and performing a number of sub-model analyses create a large amount of data, which should be sifted through to determine the 
critical stresses for all points and for all components. This is discussed in [30]. Figures 17 and 18 are samples of the result, showing one frame of the stress level of the outer metal tubes under the dynamic load during the time domain analysis.

Results for eight points around the perimeter of each component were stored in a file. Using the rain flow counting procedure [24], stress ranges and the cycle number were calculated. This process was automated so that large number of data can be handled. It should be noted that due to helical nature of components, some approximation of stress range is necessary in order to be cope with large number of data.

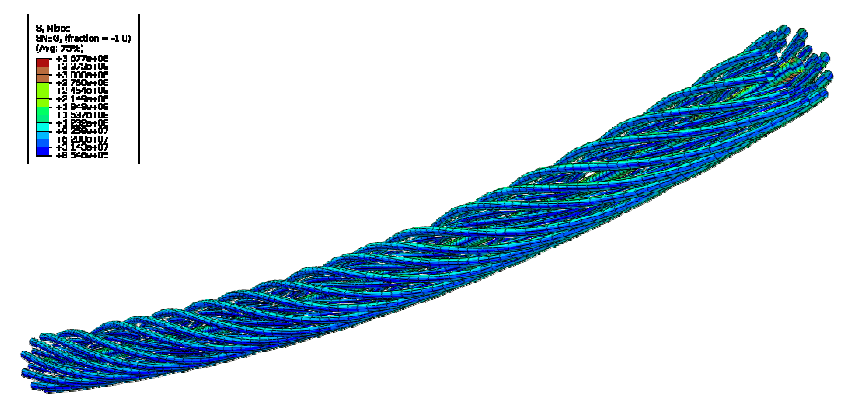

Figure 18: One frame of the time domain analysis; showing only metal tubes.

In principle there is redundancy in the umbilical makeup, so that failure of one component should not necessitate retrieving and replacing the umbilical. However, from fatigue point of view, all outer tubes (as well as inner tubes) are identical and there is no major difference between them, except if there is pressure due to the content.

The fatigue life of an umbilical requires a large number of dynamic, accounting for consistent representation of all environmental loads imposed to the structure; see e.g. [5] and [22]. For each load case the stress field over the component has to be determined. The slip between adjacent components can be ignored as the softer material used between components mitigate the effect of fretting and wear.

The calculation of the wave fatigue damage is based on $\mathrm{X}^{\prime}$ curve as specified in API RP $2 \mathrm{~A}$ and $\mathrm{E}$ S-N curve as recommended by UK-HSE:

$N=\left\{\begin{array}{c}2.5 \times 10^{13}(S C F \times \Delta \sigma)^{-3.74} \text { API } X^{\prime}-\text { Curve } \\ 1.04 \times 10^{12}(S C F \times \Delta \sigma)^{-3.0} \text { HSE E-Curve }\end{array}\right.$

$N$ is the permissible number of cycles for a stress range $\Delta \sigma$ $\mathrm{MPa}, \mathrm{SCF}$ is the stress concentration factor which is assumed to be 1.3. Results reported here are based on the HSE E curve.

A typical fatigue life distribution plot, for one outer tube, along the umbilical length is given in Figure 19. This figure give a composite results for various vessel ordination, soil condition, hydrodynamic coefficient and point around the cross section (8 points were chosen). This study used the rainflow algorithm in MATLAB code developed by Nieslony [24]. The fatigue damage is highest in touch down zone, followed by the connection point at the FPSO.

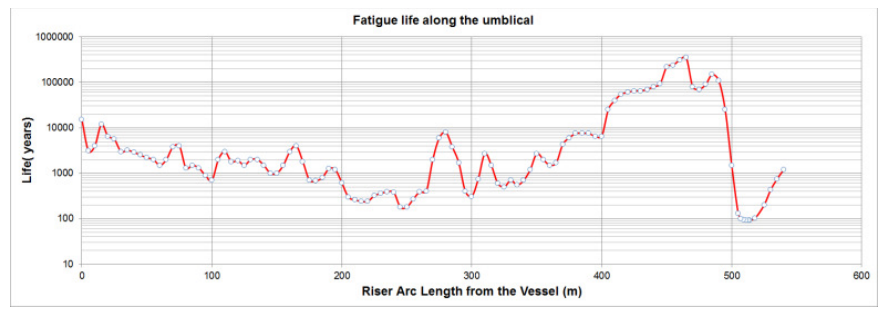

Figure 19: Fatigue lives along the umbilical length

\section{CONCLUDING REMARKS}

This paper discussed the design of dynamic umbilical with emphasis on the fatigue life estimation, using Abaqus. It is shown how to define an irregular sea state for use in Abaqus/Aqua for both strength and fatigue life calculations.

Failure modes for umbilicals range from material degradation to fatigue failure and loss of strength under extreme conditions. This is particularly the case for harsh and dynamic, environments where the loading conditions are random; see [13] and [14]. Failure is defined as an incident that is detrimental to the functionality of the fluid conduits, electrical conductor or fibre optical cable. In the past, the majority failure (about 2/3) have occurred during installation and commissioning and almost half the failures associated to manufacturing and installation errors. Most mechanical failures were associated to the failure of attachment/hang-off points.

In the sub-modelling approach, a global model is developed and its solution determined. The displacements at each node that enclose the local model's boundary are determined from the global solution. These nodal displacements are then interpolated and applied to the local model at the local model's boundary as the forced response boundary conditions. The accuracy of the local model depends on how closely the global model captures the solution at the local model's boundary. The computational savings is achieved by forcing element size consistency and by reducing the total number of elements in the global model. The success of this approach is highly dependent upon the determination of the homogenized properties used in the global analysis. The sub-modelling analysis technique in Abaqus [1] has been shown to be applicable to dynamics analysis. Sub-modelling can lead to artificially high frequency content, but If the time steps of the global analysis is not too coarse any high frequency content will be minimised, also helped by the frictional forces at the interface of components.

The global and sub-model procedures do not have to be the same. For example, the global procedure can be performed in 
ABAQUS/Standard [1] to drive a sub-modelling procedure in ABAQUS/Explicit [1] and vice versa. Both global and local analyses in this study use implicit version of Abaqus [1].

There are other types of damage, e.g. fretting or wear damage [10], which was not addressed in this study. However, based on what is reported in the literature, these types of damage are mostly prevalent in multi-layered flexible pipes. Predicting, Measuring and Implementing Friction Stresses in dynmaic analysis is performed in this study bu Abaqus's sub-modelng and conact definition. However, this issue was dealt using a different approach in [6].

\section{REFERENCES}

[ 1] Abaqus 6.12, Simulia Dasault Systems.

[2] ANSI/API Specification for Subsea Umbilicals Specification 17E $4^{\text {th }}$ ed., 2010, Effective Date: April 1, 2011

[ 3 ] Barltrop, N. D. P., and Adams, A. J.,199, “.Dynamics of Fixed Marine" , Structures, ButterworthHeinemann, Oxford

[ 4 ] Clarkston, B., Valenzuela, E., Worman, P. \& Williams, P., 2009. Frade Field Dynamic Umbilicals Design and Testing. Offshore Technology Conference.

[ 5 ] Dixon, M. \& Zhao, T., 2008, "3D Modelling Improves Deepwater Umbilical Design Dependability", Offshore Technology Conference, OTC1953.

[ 6 ] Ekeberg, K. I., Ottesen, T., Aarstein, J. \& Saevik, S., 2006, Predicting, Measuring and Implementing Friction and Bending Stresses in Dynamic Umbilical Design. Offshore Technology Conferenc, OTC 17986.

[ 7 ] DNV-RP-F401, 2012, Electrical Power Cables in Subsea Applications FEBRUARY 2012

[ 8 ] DNV-RP-F105: Free Spanning Pipelines, 2006. DNVOS-F101, 2010, Submarine Pipeline Systems

[ 9 ] DNV-RP-F109, 2010, On-Bottom Stability Design of Submarine Pipelines

[ 10 ] Fernando, U., Dodds, N., Leyland, J. \& Graham, G., 2011. Prevention of Fretting/Wear Damage In Pressure Armour Layers of Flexible Pipes Designed For High Pressure Applications. Offshore Technology Conference

[ 11] Faltinsen, O.M., 2005, Hydrodynamics of High-Speed Marine Vehicles. Cambridge University Press.

[12] Fogg, D., 2011. Umbilical Technology for Arctic Projects. Offshore Technology Conference.

[ 13 ] Gloaguen, M., Bourdillion, H., Roche, F. \& Boscal de Reals, T., 2007. Dalia Flowlines, Risers and Umbilicals. Offshore Technology Conference.

[14] Gueret, R. \& Lahiri, S., 2013. Umbilical Lay In Challenging Environmental Conditions. Society of Petroleum Engineers.

[ 15 ] Hossain, M. K., Tjok, K.-M. \& Nobahar, A., 2008. Subsea Umbilical Stability Analysis. Offshore Technology Conference.
[ 16 ] Huss. M., 2013, Notes on the modelling irregular seas in time simulations, Accessed on. http://www.mhuss.se/documents/Downloads/101129m h_Notes_IrrSea_R2.pdf, 2010. Accessed on 19/12/2013.

[ 17 ] Gallagher, D. \& Grealish, F., 2003. Steel Tube Umbilicals (STUs) - Key Issues for Deep Water Dynamic Applications. Offshore, 63(11), p. 89.

[ 18 ] Johansen, R. B., Veritas, D. N. \& Ekeberg, K. I., 2005. Subsea Umbilicals - Joint Industry Project Aiming to Revise ISO 13628-5. Offshore Technology Conference

[19] ISO 13628-5:2009 Design and Operation of Subsea Umbilicals, Part 5.

[ 20 ] Li, J., Wang, H., Zhang, K. \& You, X., 2013. FEM Model and Mechanical Behavior of Flexible Risers. International Conference on Fracture and Strength of Solids.

[21 ] Martinelli, L., Lamberti, A., Ruol, P. \& Ricci, P., 2010, "Power Umbilical for Ocean Renewable Energy Systems - Feasibility and Dynamic Response Analysis", International Conference on Ocean Energy.

[22 ] Martins, C.A. and Pesce, C.P., 2002, Simplified Procedure to Assess the Fatigue-Life of Flexible Risers, $12^{\text {th }}$ ISOPE 2002, and Japan.

[23 ] Martinez, M., Perdrizet, T. \& Probyn, I., 2010. Development of 3D Finite Element Model Of Umbilical Systems For Offshore Application

[24 ] Nieslony, A., Rainflow Counting Algorithm, MATLAB File Exchange. Accessed on 22/12/2013, http://www.mathworks.co.uk/matlabcentral/fileexchan ge/authors/7825

[25] Norsok Standard, Common Requirements, Subsea Production Control Umbilical U-CR-006Rev. 1, January 1995 Specification for Subsea Umbilicals

[ 26 ] Sævik, S. \& Ekeberg, K. I., 2002. Non-Linear Stress Analysis of Complex Umbilical Cross-Section. International Conference on Offshore Mechanics and Arctic Engineering.

[ 27 Sævik, S. \& Gjøsteen, J. K., 2012. Strength Analysis Modelling of Flexibility Umbilical Members for Marine Structures. 1st ed. s.l.:Hindawi Publishing Corporation.

[ 28 ] Sen, K. T. \& Hesar, M., 2007. Riser Soil Interaction in Soft Clay Near The Touchdown Zone. Offshore TEchnology Conference.

[29] Yasseri, S.F., Bahai, H., Bazargan, H. and Aminzadeh, A., (2010), "Prediction of safe sea-state using finite element method and artificial neural networks", Ocean Engineering 37, 200-207.

[ 30 ] Yasseri, R.F., Yasseri, S.F., Wang, B, 2014, "Extreme response of Dynamic Umbilicals in Random Waves", submitted to Abaqus Reginald Users Meeting. 